\title{
An interesting case of lip swelling!
}

\section{Evelyn Wen Yee Yap}

Yap EWY. An interesting case of lip swelling!. Malays Fam Physician. 2021;16(2);70-72. https://doi.org/10.51866/cr1130

\section{Keywords:}

Melkersson-rosenthal

syndrome, orofacial

granulomatosis,

neurocutaneous disorder

\section{Authors:}

Evelyn Yap Wen Yee

(Corresponding author)

MBBS(IMU), MRCP(UK), Adv M

Derm(UKM)

Deparment of Dermatology, Hospital

Pakar Sultanah Fatimah, Muar, Johor Malaysia

Email: Chervil_82@hotmail.com

\begin{abstract}
Melkersson-Rosenthal Syndrome (also termed "Miescher-Melkersson-Rosenthal Syndrome") or (MRS), is a rare neurocutaneous disorder characterized by the clinical triad of recurring facial nerve paralysis, swelling of one or both lips and fissural tongue. We report a patient with MelkerssonRosenthal Syndrome initially referred to Dermatology Department as angioedema. The diagnosis and treatment procedures were presented with special emphasis to the clinical features of this rare condition.
\end{abstract}

\section{Case Report}

A 70-year-old gentleman presented with persistent lip swelling and pain in the tongue for one week. He was treated as angioedema with IV hydrocortisone 100mg TDS and IV chlorpheniramine maleate $4 \mathrm{mg}$ TDS for three days, but no improvement was seen. He was otherwise well, with good cognition and fully independent in his daily living activities. Further questioning revealed a history of recurrent episodes of similar complaints for 20 years. These episodes usually lasted from a few days to as long as one month. There was no fever, bowel symptoms, cough, or constitutional symptoms. Examination revealed a swollen lower lip, which led to difficulty in closing his mouth (Figure 1). This was associated with tongue fissuring (Figure 2). He also had bilateral facial nerve weakness of the lower motor neuron. An MRI of the brain was done, which showed multiple lacunar infarcts with underlying cerebral atrophy. Chest X-ray showed normal findings. Serum calcium level was normal. Mantoux test was negative. Lip biopsy showed interface mucositis. Patient did not consent for lumbar puncture. Based on the above triad of symptoms, although the biopsy did not show classical features of non-caseating granulomas, this patient was diagnosed with Melkersson-Rosenthal Syndrome. $\mathrm{He}$ was started on oral doxycycline $100 \mathrm{mg}$ daily for three months. After three months, partial improvement was seen. There was still residual lip swelling, but the patient was able to close his mouth (Figure 3). Despite persistence of his facial paralysis and fissured tongue, he was satisfied with the results achieved. We stopped his doxycycline after three months and thereafter, he did not experience recurrences of his lip swelling after one year of follow up.

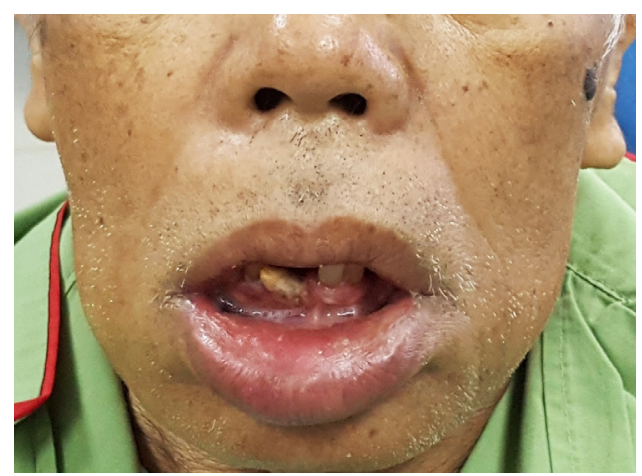

Figure 1: Swollen and everted lower lip resulting in difficulties in closing the mouth.

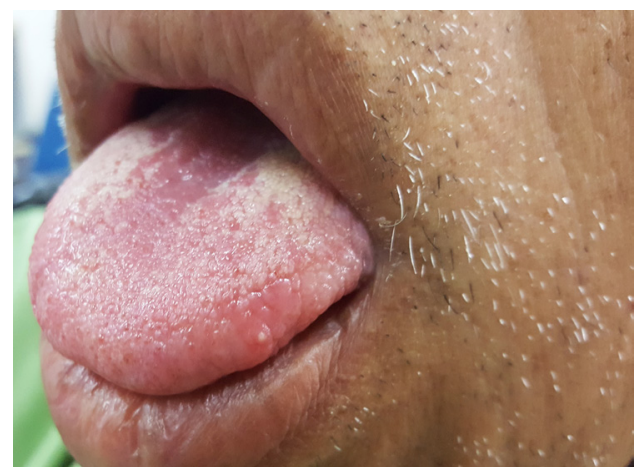

Figure 2: Fissuring seen at lateral portion of the tongue.

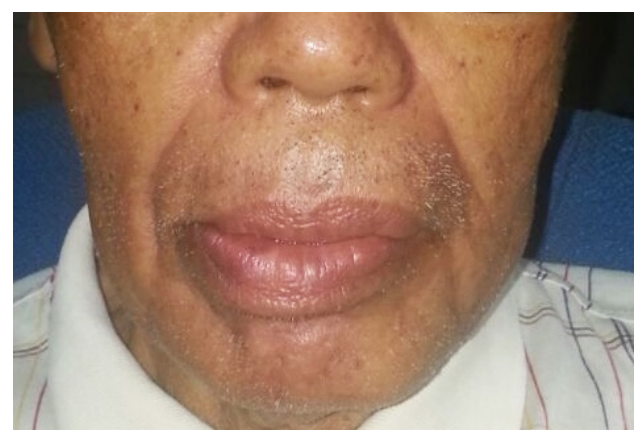

Figure 3: Partial improvement of lower lip swelling seen after three months of doxycycline. Patient was able to close mouth after treatment. 


\section{Discussion}

Melkersson-Rosenthal Syndrome (MRS) is a rare neurocutaneous disorder with an estimated incidence of $0.08 \%$ in the general population. ${ }^{1}$ It belongs to a disease entity called orofacial granulomatosis, which encompasses a spectrum of disease ranging from patients presenting with only lip swelling to patients with orofacial granulomatous inflammation, with or without recurrent facial paralysis and fissured tongue. In the case of MRS, this is characterized by the clinical triad of recurring orofacial swelling, facial nerve paralysis, and fissured tongue. This condition was first reported by Melkersson in 1928. Although etiology is unclear, various factors such as infections, genetic predisposition, immune deficiency, food intolerance, and stress have been implicated. ${ }^{2}$ Previous literature has found that MRS frequently affects those in their second and third decades of life, with incidence reducing with advancing age. ${ }^{3}$ However, our patient was not diagnosed until he was 70 years old, after suffering recurrent lip swelling for 20 years. This delay in diagnosis could be explained by the patient presenting with the oligosymptomatic version of MRS previously, making diagnosis difficult. Liu et al. reported the mean time to correct diagnosis of MRS as 9.86 years. ${ }^{2}$ The observation of the presentation of the full triad of symptoms of MRS may take a long time, sometimes even a full lifespan, since different symptoms may appear at different stages throughout the life cycle. ${ }^{4}$

We were fortunate that the patient presented with a complete symptom triad this time, which enabled the diagnosis of MRS to be made promptly. This occurred in about $8-18 \%$ of patients based on the prior literature. ${ }^{2}$ More commonly, patients present only with recurrent lip swelling. Such cases are termed cheilitis granulomatosa, and they can be mistaken for angioedema. ${ }^{5}$ To differentiate, cheilitis granulomatosa tends to be more persistent and does not improve with antihistamines. It can lead to fibrosis of involved tissues on histology, which is not seen with angioedema. ${ }^{6}$ Other differential diagnoses include oral Crohn's disease, sarcoidosis, or tuberculosis infection. In this patient, the absence of bowel symptoms at this age together with non-supportive biopsy results excluded the possibility of Crohn's disease. The normal chest X-ray with negative Mantoux and nongranulomatous histology excluded tuberculosis. Normal calcium and serum angiotensin converting enzyme levels along with an unremarkable chest X-ray also ruled out sarcoidosis. Even though the MRI of his brain showed multiple lacunar infarcts, however, these findings do not account for his bilateral facial nerve palsy of the lower motor neuron. It has been postulated that the facial nerve paralysis of MRS may be a result of pressure on the nerve passing through the facial canal within the temporal bone due to orofacial edema, or of granulomatous infiltration of the nerve.

MRS is a diagnosis made clinically. Biopsy may help in diagnosing MRS of the oligosymptomatic or monosymptomatic subtype, or to identify other possible causes of lip swelling. ${ }^{6}$ Characteristic histopathological features of MRS include lymphedema, non-caseating epithelioid cell granulomas, multinucleated Langerhans cell-type giant cells, perivascular mononuclear inflammatory infiltration, and fibrosis. ${ }^{5}$ However, the absence of classical histological features in our case does not preclude a diagnosis of MRS. Zimmer et al. reported that only $46 \%$ of MRS patients had granulomatous changes, 36\% had nonspecific inflammation, $11 \%$ had incidental findings, and $7 \%$ showed no histopathologic abnormalities. ${ }^{8}$

As the etiology is not fully elucidated, treatment of MRS is difficult. In previous published reports, no treatment was given for approximately $40 \%$ of the cases. In the remaining cases, attempts at treating this condition involved using corticosteroids (systemic and intralesional), surgery, antibiotics (minocycline, doxycycline, metronidazole), and anti-lepromatous agents (dapsone and clofazimine). Intralesional steroid injections used alone or in combination with minocycline or high-dose intravenous methylprednisolone showed a good therapeutic response. Effective surgical interventions employed included liposuction and cheiloplasty to reduce facial edema or total decompression of the facial nerve to prevent further attacks of facial paralysis. The use of tumor necrosis factoralpha (TNF-alpha) inhibitor has been associated with varying results, with some case reports showing therapeutic efficacy, though there was one report of de novo induction of MRS in a psoriatic arthritis patient treated with anti-TNF-alpha. Thalidomide was also tried with good response.?

In conclusion, MRS is a diagnosis frequently 
missed due to difficulty in diagnosis, especially when presenting in the oligosymptomatic variants. The diagnosis should be considered when a patient presents with recurrent orofacial swelling, either in isolation or in combination with other symptoms. No standard treatment is available as of yet. The rarity of this condition makes large randomized controlled trials difficult to conduct. However, in this case, we achieved satisfactory results with three months of doxycycline.

\section{How does this paper make a difference to general practice?}

- This case report highlights the importance of considering other differential diagnoses when encountering a case of lip swelling that does not improve with antihistamines.

- Associated symptoms and signs, in this case, the remitting-relapsing nature over a prolonged period, plus clinical signs of bilateral facial nerve palsy of the lower motor neuron and fissured tongue, should be sought for in order to not miss a diagnosis of Melkersson-Rosenthal Syndrome.

\section{References}

1. Hornstein OP. Melkersson-Rosenthal syndrome. A neuro-mucocutaneous disease of complex origin. Curr Probl Dermatol 1973; 5:117-56.

2. Liu R, Yu S. Melkersson-Rosenthal syndrome: a review of seven patients. Journal of clinical neuroscience. 2013 Jul 1;20(7):993-5.

3. Dodi I, Verri R, Brevi B, Bonetti L, Balestrier A, Saracino A, Akamin R, Izzi GC, Vanelli M, Sesenna E. A monosymptomatic MelkerssonRosenthal syndrome in an 8-year old boy. Acta bio-medica: Atenei Parmensis. 2006 Apr 1;77(1):20-3.
4. Elias MK, Mateen FJ, Weiler CR. The Melkersson-Rosenthal Syndrome: A retrospective study of biopsied cases. J Neurol 2013; 260: 138-43.

5. Pisanty S, Sharav Y. The melkersson-rosenthal syndrome. Oral Surgery, Oral Medicine, Oral Pathology. 1969 Jun 1;27(6):729-33.

6. Shapiro M, Peters S, Spinelli HM. Melkersson-Rosenthal syndrome in the periocular area: a review of the literature and case report. Annals of plastic surgery. 2003 Jun 1;50(6):644-8.
7. Sciubba JJ, Said-Al-Naief N. Orofacial granulomatosis: presentation, pathology and management of 13 cases. Journal of oral pathology \& medicine. 2003 Nov;32(10):57685.

8. Zimmer WM, Rogers III RS, Reeve CM, Sheridan PJ. Orofacial manifestations of Melkersson-Rosenthal syndrome: a study of 42 patients and review of 220 cases from the literature. Oral surgery, oral medicine, oral pathology. 1992 Nov 1;74(5):610-9.

9. Wehl G, Rauchenzauner M. A systematic review of the literature of the three related disease entities cheilitis granulomatosa, orofacial granulomatosis and MelkerssonRosenthal syndrome. Current pediatric reviews. 2018 Aug 1;14(3):196-203. 